\title{
SUR L'EFFET DU TRAITEMENT \\ PAR LES RAYONS ULTRAVIOLETS \\ DES EAUX UTILISEES EN INDUSTRIE LAITIERE
}

\author{
par \\ Jacques CASALIS, François M. LUQUET, \\ Françoise FONTAINE et Jean DUMONT \\ (Laboratoire d'industrie laitière de l'Ecole nationale supérieure \\ des Industries agricoles et alimentaires.)
}

Ayant eu connaissance des essais effectués d'avril à octobre 1965 par M. Coutris, directeur du Service de contrôle des eaux de la ville de Paris, sur le traitement des eaux destinées à la consommation humaine par le procédé d'actinisation de M. W. P. de Stoutz, nous avons été conduits à rechercher si ce traitement pouvait s'appliquer utilement aux eaux utilisées en industrie laitière. A cet égard, il paraît opportun de rappeler que les critères de la qualité bactériologique de l'eau sont différents suivant que cette dernière est destinée à la consommation humaine ou à l'utilisation dans la fabrication de produits laitiers : l'eau potable doit être exempte de microorganismes pathogènes susceptibles de nuire à la santé du consommateur ; l'eau utilisée dans la fabrication des produits laitiers doit répondre à ces mêmes exigences, mais en outre être dépourvue de germes technologiquement redoutables et susceptibles de provoquer des accidents de fabrication en beurrerie, caséinerie et fromagerie.

Sans entrer dans le détail de leurs actions nocives, et en précisant que, suivant le but poursuivi, ces microbes peuvent être ou non indésirables, on peut faire entrer dans la catégorie des germes. technologiquement dangereux :
a) les levures,
b) les moisissures,
c) les bactéries coliformes,
d) les thermorésistants,
e) les sporulés.

S'il est possible de détruire, par les procédés classiques de pasteurisation, les levures, les moisissures et les bactéries coliformes, l'industrie laitière se trouve jusqu'à présent désarmée devant une contamination par les thermorésistants et surtout par les sporulés, 
qui ne peuvent être éliminés que par une stérilisation difficilement applicable dans la pratique. C'est donc les bactéries sporulées, et en particulier le genre " clostridium ", qui ont fait l'objet essentiel de cette étude.

Appareil utilisó (fig. 1).

Le liquide à traiter circule à l'abri de l'air, à l'intérieur d'un faisceau de tubes en matière perméable aux rayonnements ultraviolets de courte longueur d'onde.

L'appareil d'expérience comporte deux faisceaux de cellules pouvant être branchées soit en parallèle, soit en série.

a) En parallèle: le débit est de l'ordre de 500 litres/heure.

b) En série: le débit est de l'ordre de 250 litres/heure (capacité nominale de l'appareil).

Les rayonnements ultra-violets sont émis par des lampes à vapeur de mercure dont les longueurs d'onde sont pour 95 p. 100 de l'émission, situées dans les lignes de résonance du mercurs, soit $2537 \mathrm{~A}^{\circ}$.

Ces brûleurs sont alimentés par des transformateurs à haute tension, commandés par des contacteurs comportant 3 positions différentes, correspondant à 3 intensités d'irradiation qui ellesmêmes peuvent varier en fonction du temps, suivant le montage, soit en parallèle, soit en série, des faisceaux tubulaires.

Cette disposition et ce système de réglage ont permis de faire varier les intensités au cours des expériences auxquelles nous avons procédé, selon tableau I :

TABLEAU I

TEMPS D'IRRADIATION EN SECONDES

SUIVANT LE MONTAGE DE L'APPAREIL

\begin{tabular}{|c|c|c|c|}
\hline Intensités d'irradiation & $U V 1$ & $U V 2$ & $U V 3$ \\
\hline $\begin{array}{l}\text { Montage en série } \ldots \ldots \ldots \ldots \\
\text { Montage en parallèle } \ldots \ldots \ldots\end{array}$ & $\begin{array}{l}3 \text { s } 47 \\
1 \text { s } 73\end{array}$ & $\begin{array}{l}6 \text { s } 94 \\
3 \text { s } 47\end{array}$ & $\begin{aligned} 10 & \text { s } 4 \\
5 & \text { s } 2\end{aligned}$ \\
\hline
\end{tabular}

\section{Conditions générales des essais}

Avant chaque essai, l'appareil a été nettoyé et désinfecté suivant les indications du constructeur, en y faisant circuler successivement et en circuit fermé : 
a) pendant 15 minutes une solution à $60^{\circ} \mathrm{C}$ de soude à $3 \mathrm{p} .100$;

b) pendant 3 minutes de l'eau froide pour éliminer la solution sodique ;

c) pendant 15 minutes une solution à $60^{\circ} \mathrm{C}$ d'acide nitrique à 4,5 p. 100 ;

d) pendant 3 minutes de l'eau froide pour éliminer la solution acide ;

e) pendant 15 minutes une solution à $60^{\circ} \mathrm{C}$ d'hypochlorite de soude à 2,5 p. 100 ;

f) pendant 3 minutes de l'eau froide, de manière à obtenir un rinçage final.

Bien entendu, au cours des premiers essais des numérations microbiennes ont été effectuées après les opérations de nettoyage et ont permis de contrôler l'efficacité de la technique employée.

\section{Origine des eaux soumises au traitement}

Dans les différentes séries d'expérience, nous avons utilisé tantôt de l'eau prélevée dans la Scarpe, rivière qui traverse Douai et dans laquelle se déverse une partie des égouts de la ville, cette eau ayant été traitée soit telle quelle, soit après enrichissement en différentes espèces microbiennes, tantôt de l'eau du réseau de distribution d'eau potable de la ville de Douai, celle-ci ayant été préalablement ensemencée avec les différentes espèces microbiennes mentionnées dans le tableau II. Nous nous sommes ainsi volontairement placés dans des conditions de travail beaucoup plus sévères que celles de l'industrie laitière qui utilise des eaux provenants de puits ou de réseaux de distribution.

Outre les différentes souches mentionnées au tableau II et qui, en raison de leur résistance particulière aux antiseptiques et aux traitements thermiques ont fait plus spécialement l'objet de nos déterminations, nous avons également cherché à vérifier l'action des rayons ultraviolets sur d'autres espèces telles que Coliformes, Escherichia Coli, Caséolytiques, Streptocoques fécaux et en général sur la flore saprophyte mésophile ou psychrotrophe.

Pour le dénombrement de ces différentes espèces microbiennes, les milieux de culture suivants ont été employés :

- Pour les Clostridia glucidolytiques :

- Milieu tryptone T 65 ;

- Milieu Soytone S 65 ;

- Milieu VL-VF* en tubes profonds.

*(viande levure-viande foie) 
SOUCHES MICROBIENNES UTILISÉES AU COURS DES ESSAIS

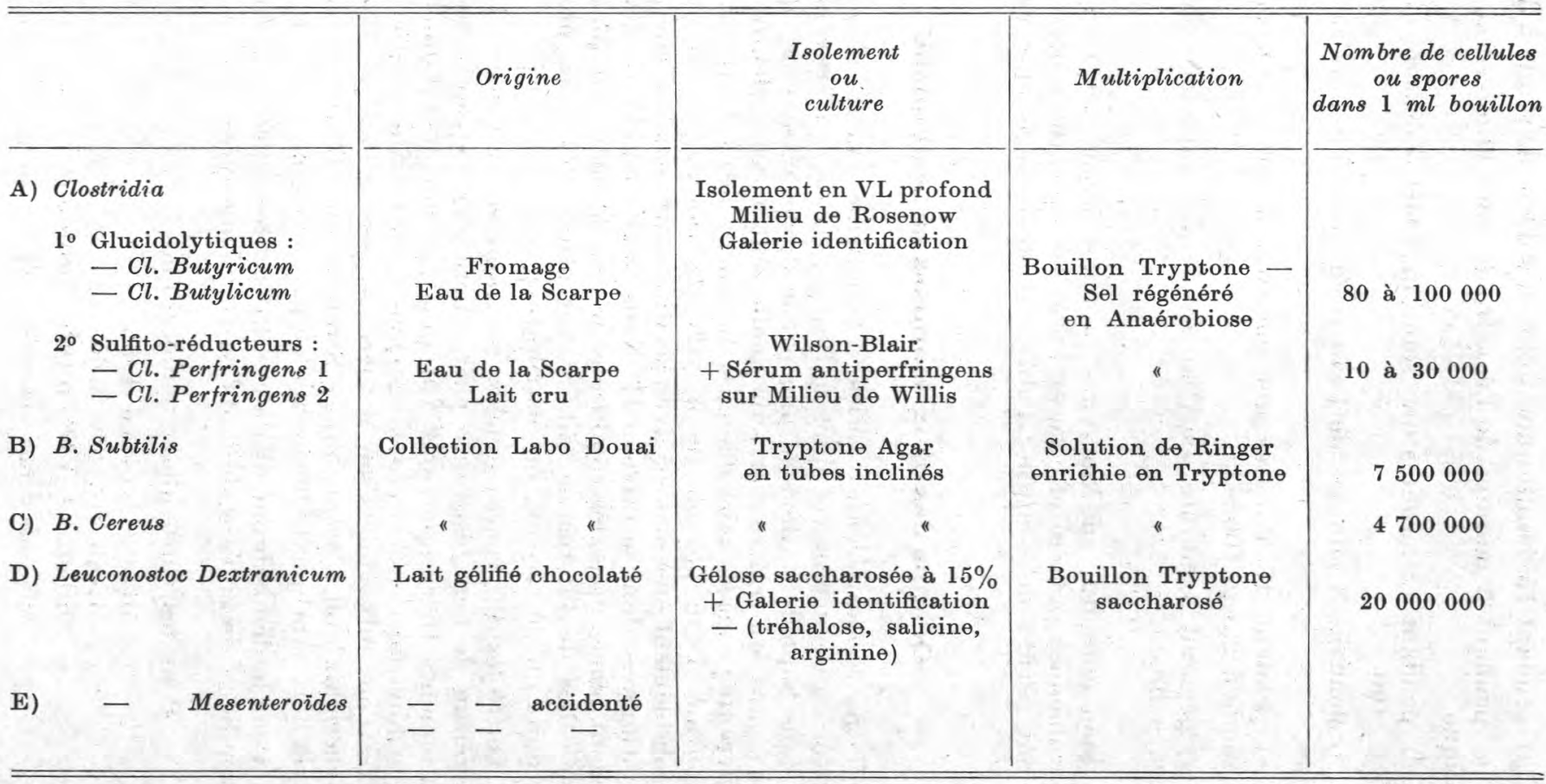


- Pour les Clostridia sulfito-réducteurs :

- Milieu de Wilson-Blair (Alun de Fe) ;

- Milieu VL au sulfite de Na à 0,25 p. 100 ;

- Milieu de Willis.

- Pour le B. Subtilis et le B. Cereus :

- Gélose nutritive + tests complémentaires (lécithine, type respiratoire, etc.).

- Pour les Leuconostocs :

- Gélose saccharosée à 15 p. 100.

- Pour les Coliformes :

- Milieu au désoxycholate lactose Agar ;

- Bouillon lactosé bilié au vert brillant.

Colimétrie sur membrane :

- Milieu de Chapman au T.T.C. (triphényl-tétrazoliumchlorure) ;

- Milieu Endo.

- Pour Escherichia Coli :

- Bouillon lactosé bilié au vert-brillant;

- Eau peptonée ;

- Test de Mackenzie avec tests complémentaires

- Pour les caséolytiques :

- Gélose au caséinate de chaux.

- Pour les streptocoques fécaux :

- Test présomptif : milieu de Rothe;

- Test confirmatif : milieu de Litsky;

- Milieu de Barnes au T.T.C.

- Milieu au tellurite de potassium.

- Pour la flore totale aérobie :

- Gélose nutritive.

\section{Influence de différents facteurs dans la stérilisation par les U.V.}

\section{Température:}

Il paraît opportun de préciser que le traitement par les ultraviolets auxquels les liquides ont été soumis, n'a qu'une influence très faible sur la température de ces liquides. Nous avons constaté des variations de l'ordre de 3 à $5^{\circ} \mathrm{C}$ entre la température du liquide 
à l'entrée dans l'appareil et à la sortie. La température des liquides dans les conditions de nos essais était de 13 à $15^{\circ} \mathrm{C}$ à l'entrée de l'appareil et de 15 à $18^{\circ} \mathrm{C}$ à la sortie. Ces variations de température sont de trop peu d'importance pour que l'on puisse leur attribuer une influence sur la destruction des germes. C'est done à la seule action des rayons ultraviolets que sont dues les actions germicides constatées dans nos expériences, dont les résultats sont résumés dans les tableaux III, IV et V.

\section{TABLEAU III}

TRATTEMENT DE L'EAU DE LA SCARPE

(nombre de germes dans $100 \mathrm{ml}$ )

\begin{tabular}{|c|c|c|c|c|c|c|c|}
\hline \multirow{2}{*}{ Essai } & \multirow{2}{*}{ Débit } & \multirow{2}{*}{$\begin{array}{l}\text { Tempé- } \\
\text { rature } \\
\text { Entrée } \\
\text { Sortie }\end{array}$} & \multirow{2}{*}{$\begin{array}{l}\text { Quan- } \\
\text { tité } \\
\text { traitée }\end{array}$} & \multicolumn{2}{|c|}{$\begin{array}{l}\text { Clostridium } \\
\text { Butyricum }\end{array}$} & \multicolumn{2}{|c|}{$\begin{array}{l}\text { Clostridium } \\
\text { Perfringens }\end{array}$} \\
\hline & & & & Entrée & Sortie & Entrée & Sortie \\
\hline 326 & $215 \mathrm{l} / \mathrm{h}$ & $11^{\circ}$ & 501 & 1000 & 0 & 1000 & 0 \\
\hline 826 & $200 \mathrm{l} / \mathrm{h}$ & $12^{\circ}$ & 501 & 0 & 0 & 200 & 0 \\
\hline I 426 & $240 \mathrm{l} / \mathrm{h}$ & $9^{\circ}$ & 501 & 0 & 0 & 1000 & 0 \\
\hline 2326 & $240 \mathrm{l} / \mathrm{h}$ & $9^{\circ}$ & 501 & 6000 & 0 & 100 & 0 \\
\hline 2526 & $240 \mathrm{l} / \mathrm{h}$ & $9^{\circ}$ & 501 & 1000 & 0 & 100 & 0 \\
\hline 2526 & $240 \mathrm{l} / \mathrm{h}$ & $11^{\circ}$ & 251 & 1000 & 0 & 100 & 0 \\
\hline
\end{tabular}

\section{$2^{\circ} \mathrm{DBO}$ (demande biologique en oxygène) :}

Comme le montre le tableau VII, qui est un résumé des essais, la DBO (mesurée directement avec un analyseur Beckman, modèle 777) varie légèrement et a tendance à augmenter de 2,8 p. 100 dans une eau assez chargée biologiquement. Par contre, dans une eau épurée et traitée, telle une eau de ville, elle augmente de 2,5 p. 100.

\section{$3^{\circ}$ Matières organiques et temps d'action:}

Différents essais ont été effectués afin de rechercher le taux de matières organiques à partir duquel, pour un temps donné d'irradiation, l'action des U. V. se révèle inefficace.

Le tableau VI montre qu'en faisant varier le temps du simple au double pour un taux de matières organiques donné, 1'on obtenait des résultats intéressants. Dans le cas d'utilisation de l'appareil selon les normes du constructeur, il semblerait que le taux limite soit de 5 gr. par litre. 
TABLEAU IV

(nombre de germes exprimés pour $100 \mathrm{ml}$ )

\begin{tabular}{|c|c|c|c|c|c|c|c|c|}
\hline \multirow[t]{2}{*}{ No Essai } & \multirow{2}{*}{$\begin{array}{l}\text { Temps } \\
\text { de } \\
\text { passage }\end{array}$} & \multirow{2}{*}{$\begin{array}{l}\text { Taux } \\
\text { de matières } \\
\text { organiques }\end{array}$} & \multicolumn{2}{|c|}{ Flore totale Aérobie } & \multicolumn{2}{|c|}{$\begin{array}{c}\text { Clostridium } \\
\text { du groupe Butyrique }\end{array}$} & \multicolumn{2}{|c|}{ Clostridium Perfringens } \\
\hline & & & Entrée & Sortie & Entrée & Sortie & Entrée & Sortie \\
\hline A & $10 \mathrm{~s}$ & - & 一 & - & 3300 & 0 & 500 & - \\
\hline 3 & $6 \mathrm{~s} 5$ & $21 \mathrm{mg} / \mathrm{l}$ & 7280000 & 100 & - & - & - & - \\
\hline 2 & $5 \mathrm{~s}$ & $11,20 \mathrm{mg} / \mathrm{l}$ & 54000000 & 400 & 1200 & 30 & 133 & 13 \\
\hline 1 & $6 \mathrm{~s}$ & $11,42 \mathrm{mg} / \mathrm{l}$ & 7000000 & 600 & 1500 & 0 & 128 & 4 \\
\hline 19 & $7 \mathrm{~s}$ & $42,50 \mathrm{mg} / \mathrm{l}$ & 86000000 & 800 & 47000 & 200 & 8800 & 0 \\
\hline 20 & $8 \mathrm{~s}$ & $42,50 \mathrm{mg} / \mathrm{l}$ & 86000000 & 200 & 47000 & 0 & 8800 & 0 \\
\hline $\mathrm{X} 1$ & $10 \mathrm{~s}$ & $10 \mathrm{mg} / 1$ & - & - & 6000 & 0 & 100 & 0 \\
\hline $\mathrm{X}_{2}$ & $12 \mathrm{~s}$ & $\mathrm{mg} / \mathrm{l}$ & - & - & 1000 & 0 & 1000 & 0 \\
\hline 12 & $22 \mathrm{~s}$ & $\mathrm{mg} / \mathrm{l}$ & 2700000 & 0 & $800 c 0$ & 0 & 14000 & 0 \\
\hline
\end{tabular}

TABLEAU V

(nombre de germes exprimés dans $100 \mathrm{ml}$ )

\begin{tabular}{|c|c|c|c|c|c|c|c|c|c|}
\hline \multirow{2}{*}{ No Essai } & \multirow{2}{*}{$\begin{array}{l}\text { Temps } \\
\text { de } \\
\text { passage }\end{array}$} & \multirow{2}{*}{$\begin{array}{c}\text { Taux } \\
\text { de matières } \\
\text { organiques }\end{array}$} & \multirow{2}{*}{$p \mathbf{H}$} & \multicolumn{2}{|c|}{ B. Subtilis } & \multicolumn{2}{|c|}{ B. Cereus } & \multicolumn{2}{|c|}{ Leuconostoc } \\
\hline & & & & Entrée & Sortie & Entrée & Sortie & Entrée & Sortie \\
\hline 3 & $6 \mathrm{~s} 5$ & $21 \mathrm{mg} / 1$ & $6,3-6,8$ & 52500000 & 0 & - & 一 & - & - \\
\hline 4 & $7 \mathrm{~s}$ & $20 \mathrm{mg} / 1$ & $6,3-6,8$ & - & - & 32900000 & 750 & - & - \\
\hline 5 & $6 \mathrm{~s} 5$ & $20 \mathrm{mg} / \mathrm{l}$ & $6,3-6,8$ & - & - & - & - & 35000000 & 0 \\
\hline 19 & $7 \mathrm{~s}$ & $42,50 \mathrm{mg} / 1$ & 6,25 & 14000000 & 0 & 72000000 & 800 & 110000 & 0 \\
\hline 20 & $8 \mathrm{~s}$ & $42,50 \mathrm{mg} / \mathrm{l}$ & 6,25 & 14000000 & 0 & 72000000 & 0 & 110000 & 0 \\
\hline
\end{tabular}


TABLEAU

(nombre de germes exprimés

\begin{tabular}{|c|c|c|c|c|c|c|c|c|}
\hline \multirow{2}{*}{ Essai } & \multirow{2}{*}{$\begin{array}{l}\text { Temps } \\
\text { de } \\
\text { passage }\end{array}$} & \multirow{2}{*}{$\begin{array}{c}\text { Taux } \\
\text { de matières } \\
\text { organiques } \\
\text { dans } \\
100 \mathrm{ml} \\
(\text { en } \mathrm{g})\end{array}$} & \multirow{2}{*}{$\begin{array}{c}\text { Extrait } \\
\text { sec } \\
\text { dans } \\
100 \mathrm{ml} \\
(\text { en } \mathrm{g})\end{array}$} & \multirow{2}{*}{$p \mathrm{H}$} & \multicolumn{2}{|c|}{ Flore totale aérobie } & \multicolumn{2}{|c|}{ Germes coliformes } \\
\hline & & & & & Entrée & Sortie & Entrée & Sortie \\
\hline 14 & $10 \mathrm{~s} 5$ & 1,180 & 0,278 & 5,8 & 2700000 & 300 & 10000 & 0 \\
\hline 13 & $11 \mathrm{~s}$ & 1,400 & 0,307 & 5,5 & 3200000 & 6000 & 25000 & 5 \\
\hline 15 & 8 \& 5 & 2,040 & 0,488 & 5,4 & 64000000 & 800 & 60000 & 0 \\
\hline 16 & 17 s 5 & 2,040 & 0,488 & 5,4 & 64000000 & 0 & 60000 & 0 \\
\hline 17 & $9 \mathrm{~s}$ & 3,120 & 0860 & 4,75 & 32000000 & 950 & 25000 & 50 \\
\hline 18 & $20 \mathrm{~s}$ & 3,120 & 0,860 & 4,75 & 32000000 & 0 & 25000 & 0 \\
\hline 21 & 8 s 6 & 5,520 & 2,07 & 5,45 & 62400000 & 5500 & 2500 & 0 \\
\hline 22 & $22 \mathrm{~s}$ & 5,520 & 2,07 & 5,45 & 62400000 & 0 & 2500 & 0 \\
\hline
\end{tabular}

TABLEAU VII

(DBO exprimée en mg d'oxygène/l)

\begin{tabular}{|c|c|c|c|c|c|}
\hline \multirow{2}{*}{$\begin{array}{c}\text { Nature } \\
\text { du liquide }\end{array}$} & \multirow{2}{*}{$\begin{array}{l}\text { Temps } \\
\text { de } \\
\text { passage }\end{array}$} & \multicolumn{2}{|c|}{$D B O$} & \multicolumn{2}{|c|}{$p \mathrm{H}$} \\
\hline & & Entrée & Sortie & Entrée & Sortie \\
\hline $\begin{array}{c}\text { Eau de la Scarpe } \\
\| \\
\|\end{array}$ & $\begin{array}{l}5 \mathrm{~s} \\
6 \mathrm{~s} \\
6 \mathrm{~s} 5\end{array}$ & $\begin{array}{l}6,8 \\
7,9 \\
7,95\end{array}$ & $\begin{array}{l}7,05 \\
8,5 \\
7,5\end{array}$ & $\begin{array}{l}7,35 \\
7,25 \\
7,40\end{array}$ & $\begin{array}{l}7,25 \\
7,40 \\
7,45\end{array}$ \\
\hline $\begin{array}{c}\text { Eau de la Ville } \\
\Downarrow " \\
«\end{array}$ & $\begin{array}{l}10 \mathrm{~s} \\
11 \cdot \mathrm{s} \\
18 \mathrm{~s}\end{array}$ & $\begin{array}{l}5,40 \\
5,40 \\
5,40\end{array}$ & $\begin{array}{l}5,60 \\
5,95 \\
5,70\end{array}$ & $\begin{array}{l}6,7 \\
6,7 \\
6,7\end{array}$ & $\begin{array}{l}6,75 \\
6,7 \\
6,75\end{array}$ \\
\hline
\end{tabular}


VI

pour $100 \mathrm{ml}$ de liquide)

\begin{tabular}{|c|c|c|c|c|c|c|c|}
\hline \multicolumn{2}{|c|}{$\begin{array}{c}\text { Germes } \\
\text { Coliformes-indologènes }\end{array}$} & \multicolumn{2}{|c|}{ Germes caséolytiques } & \multicolumn{2}{|c|}{ Clostridium butyricum } & \multicolumn{2}{|c|}{ Clostridium perfringens } \\
\hline Entrée & Sortie & Entrée & Sortie & Entrée & Sortie & Entrée & Sortie \\
\hline 10000 & 0 & 3000000 & 200 & 400000 & 0 & 76000 & 0 \\
\hline 25000 & 0 & 600000 & 1300 & 250000 & 0 & 43000 & 20 \\
\hline+ de 10000 & 0 & 1200000 & 800 & 190000 & 0 & 7500 & 0 \\
\hline + de 10000 & 0 & 1200000 & 0 & 190000 & 0 & 7500 & 0 \\
\hline+ de 10000 & 0 & 400000 & 0 & 80000 & 100 & 140000 & 400 \\
\hline+ de 10000 & 0 & 400000 & 0 & 80000 & 0 & 140000 & 0 \\
\hline 250 & 0 & 660000 & 1500 & 9000 & 0 & 4300 & 0 \\
\hline 250 & 0 & 660000 & 0 & 9000 & 0 & 4300 & 0 \\
\hline
\end{tabular}

\section{Conclusions}

Les résultats du traitement des eaux par les rayons ultraviolets dans les conditions de nos expériences sont tout à fait remarquables: dans des eaux d'une teneur en matières organiques relativement élevée (jusqu'à $45 \mathrm{mg}$ par litre), un temps d'exposition de 8" aux rayons ultraviolets permet de détruire la totalité des germes, y compris les germes sporulés, dans un appareil d'un débit de 250 litres/heure.

Nous nous proposons de vérifier, à l'aide d'un appareil à grand débit, si ces résultats sont transposables aux conditions de travail de l'Industrie laitière, c'est-à-dire avec des appareils travaillant en continu et à des débits de plusieurs milliers de litres à l'heure. 


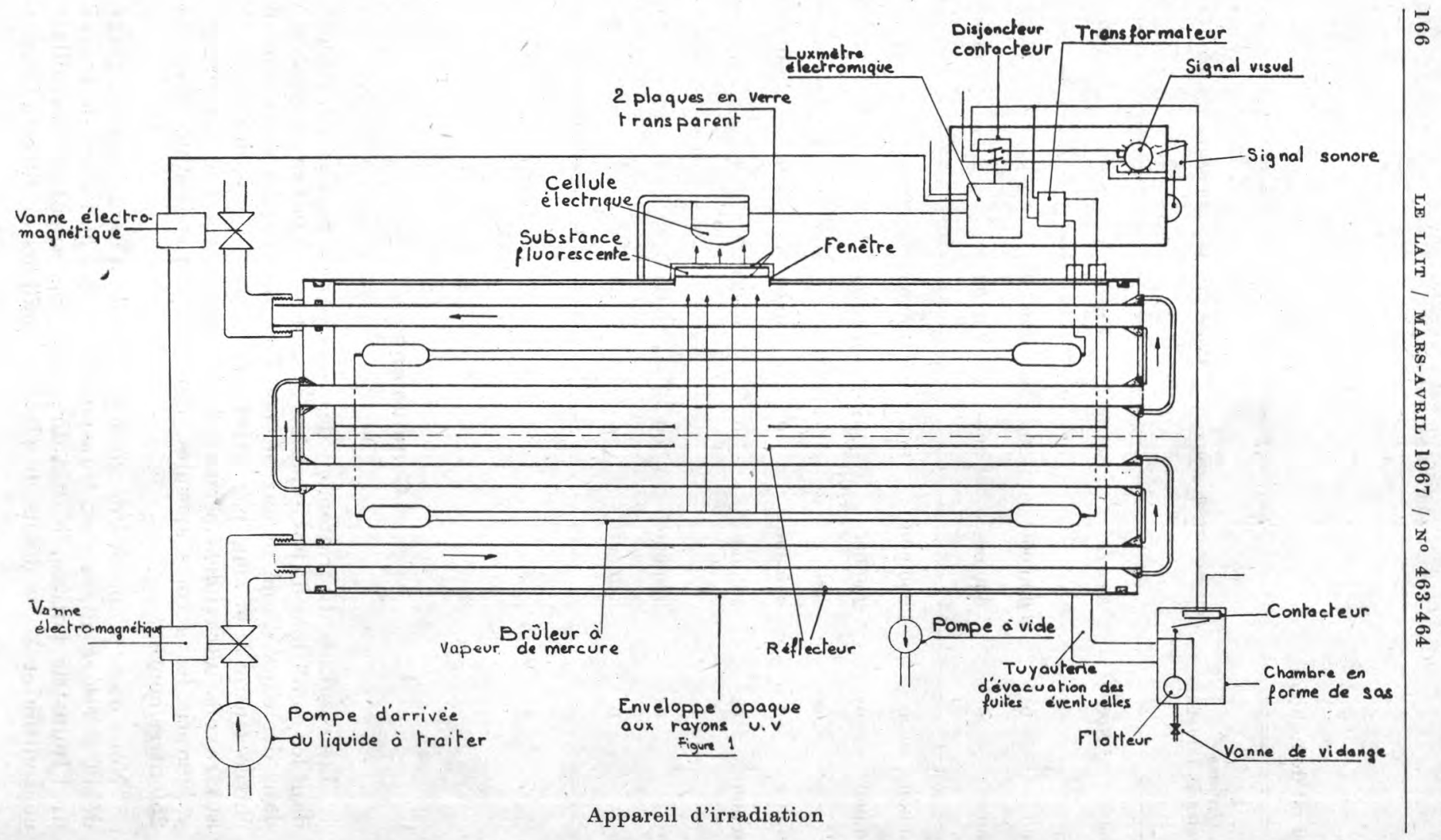

